Research Article

\title{
The Effect and Mechanism of lncRNA NR2F1-As1/miR-493-5p/ MAP3K2 Axis in the Progression of Gastric Cancer
}

\author{
Xiaobin Liao $\mathbb{D}^{1},{ }^{1}$ Linbao Wen $\mathbb{D}^{1},{ }^{2}$ and Liqiong Luo $\mathbb{1}^{3}$ \\ ${ }^{1}$ Department of General Surgery, South Hospital of Tongchuan People's Hospital, Tongchuan 727100, China \\ ${ }^{2}$ Department of Neurosurgery, The Second Affiliated Hospital of Guizhou University of Traditional Chinese Medicine, \\ Guiyang 550003, China \\ ${ }^{3}$ Department of Oncology, Lanzhou New District First People's Hospital, Lanzhou 730300, China
}

Correspondence should be addressed to Liqiong Luo; luoliqiong@lzdyyy.cn

Received 8 June 2021; Accepted 2 July 2021; Published 14 July 2021

Academic Editor: Muhammad Wasim Khan

Copyright (c) 2021 Xiaobin Liao et al. This is an open access article distributed under the Creative Commons Attribution License, which permits unrestricted use, distribution, and reproduction in any medium, provided the original work is properly cited.

\begin{abstract}
Background. LncRNA NR2F1-AS1 has been identified as an oncogene in some human tumors, such as breast cancer, nonsmall cell lung cancer, and esophageal squamous cell carcinoma. Nonetheless, whether NR2F1-AS1 is involved in the progression of gastric cancer (GC) remains unknown. Methods. The expression patterns of NR2F1-AS1, MAP3K2, and miR-493-5p in GC tissues and cells were detected by RT-qPCR. The protein expression of MAP3K2 was assessed by the Western blotting assay. The MTT assay and flow cytometry were performed to measure cell proliferation and cell apoptosis in GC cells. The transwell assay was adopted to assess cell migration in GC cells. The relationship between NR2F1-AS1, MAP3K2, and miR-493-5p was verified by a dualluciferase reporter assay. Results. The increased NR2F1-AS1 and MAP3K2 expressions were discovered in GC tissues and cells compared with control groups. Knockdown of NR2F1-AS1 and MAP3K2 dramatically suppressed cell proliferation and migration, while it enhanced cell apoptosis in GC cells. In addition, NR2F1-AS1 was found to be a sponge of miR-493-5p, and MAP3K2 was a downstream gene of miR-493-5p. Moreover, the expression of MAP3K2 was notably reduced by miR-493-5p, and NR2F1-AS1 counteracted the inhibition of miR-493-5p. Conclusion. Thus, NR2F1-AS1 was verified to regulate GC cell progression by sponging miR-493-5p to upregulate MAP3K2 expression.
\end{abstract}

\section{Introduction}

Gastric cancer (GC) is a malignant tumor derived from the epithelium of the gastric mucosal epithelium. GC accounts for the third in all malignant tumors, the first among gastrointestinal cancer, and $95 \%$ of gastric malignancies. The morbidity and mortality of GC are the third and fifth among human malignant tumors, respectively [1]. In view of the high morbidity and mortality of GC, the study on the etiology of GC has attracted many scholars. Studies have shown that obesity, gastroesophageal reflux, unreasonable diet, Helicobacter pylori infection, and atrophic gastritis are all high-risk factors for GC [2-4]. At present, surgical resection of the tumor is still the main method for the treatment of GC in clinical practice [5]. But the recurrence rate of GC patients after surgery is high, leading to a poor prognosis [6]. The key of targeted therapy is to find the genes and proteins that play a key role in the development of GC and to intervene against them, so as to achieve the purpose of treatment. At present, the targeted therapy of GC has made a substantial progress $[7,8]$.

LncRNAs regulate gene expression at multiple levels, including changing chromatin status, binding to miRNAs and mRNAs to regulate genes' expression, and binding to RNA-binding proteins to mediate intracellular signaling $[9,10]$. In recent years, great progress has been made in the research on the relationship between IncRNAs and tumor development and metastasis [11, 12]. H19 was negatively correlated with the prognosis of GC and played the carcinogenic role by regulating miRNAs and genes in GC [13-15]. PINT was found to suppress tumor progression by the crosstalk with miR-21 in GC [16]. LncRNA NR2F1-AS1 
was found to play a special role in some cancers. NR2F1-AS1 was found to accelerate cell angiogenesis in breast cancer and was associated with breast cancer recurrence $[17,18]$. In addition, previous research studies have verified that NR2F1-AS1 functions as an oncogene in nonsmall cell lung cancer, endometrial cancer, and esophageal squamous cell carcinoma [19-21]. On the contrary, NR2F1-AS1 suppressed cell growth by modulating miR-371-3p/TOB1 axis in colorectal cancer [22]. However, the information on the effect of NR2F1-AS1 in GC is limited.

In the current article, we first examined the expression levels of NR2F1-AS1 and MAP3K2 in GC and explored their effect on cell proliferation, cell apoptosis, and cell migration in GC. Furthermore, the regulatory network of NR2F1-AS1/ miR-493-5p/MAP3K2 in GC was also explored.

\section{Materials and Methods}

2.1. Patients and Clinical Tissues Samples. The tissue samples of 44 GC patients at South Hospital of Tongchuan People's Hospital were collected for our experiments. Specimens from each patient included GC tissues and matched distal nontumor tissues. Before surgery, none of the patients had received any preoperative radiotherapy, chemotherapy, or immunotherapy. This study was approved by the Ethics Committee of South Hospital of Tongchuan People's Hospital. The patients or their families have signed the informed consent.

2.2. Cell Lines. Human normal gastric mucosa epithelial cell line (GES-1) and GC cell lines (HGC-27, AGS, MKN-74, and SNU-5) were obtained from the Typical Cell Bank of Chines Academy of Sciences (Shanghai, China). The cells were cultured in the Roswell Park Memorial Institute 1640 (RPMI-1640) medium containing 10\% foetal bovine serum (FBS), penicillin, and streptomycin. The culture atmosphere of the cell incubator was $37^{\circ} \mathrm{C}, 5 \% \mathrm{CO}_{2}$, and $95 \%$ humidity. Cell passage was carried out when the cell confluence reached $80 \%$.

2.3. RT-qPCR. Total RNA was obtained from GC tissues or cells by using TRIzol. Then, the cDNA was synthesized by using a reverse transcription kit. $2 \mu \mathrm{l}$ reverse transcription product was taken for PCR reaction. The PCR reaction system was SYBR Green Master Mix $10 \mu \mathrm{L}$, forward and reverse primers $0.8 \mu \mathrm{L}$ each, cDNA $3 \mu \mathrm{L}$, and $\mathrm{ddH}_{2} \mathrm{O} 7.4 \mu \mathrm{L}$. Reaction conditions were $95^{\circ} \mathrm{C}$ for $2 \mathrm{~min} ; 95^{\circ} \mathrm{C}$ for $15 \mathrm{~s}, 60^{\circ} \mathrm{C}$ for $30 \mathrm{~s}, 72^{\circ} \mathrm{C}$ for $30 \mathrm{~s}$, and a total of 40 cycles. GAPDH was used as an internal reference for NR2F1-AS1 and MAP3K2, while U6 was used as an internal reference for miR-493-5p. The relative expressions of NR2F1-AS1, MAP3K2, and miR$493-5 p$ were calculated by the $2^{-\triangle \triangle C T}$ method. Primer sequences are given in Table 1.

2.4. Cell Transfection. AGS and MKN-74 cells were selected for the functional experiments. NR2F1-AS1 si-RNA (5'UAAUAGAAAUAUUGAGAACAU-3'), MAP3K2 si-RNA,
Table 1: Primers sequences.

\begin{tabular}{lcc}
\hline Gene & & Primer sequences \\
\hline NR2F1- & Forward & $5^{\prime}$ - CAGCGGTGCAAACCATGTGC-3' \\
AS1 & Reverse & $5^{\prime}$ - GCAAGTTGGCTGAACCAAATG-3' \\
\hline miR-493- & Forward & $5^{\prime}$ - TCCTACGGAGAGGCTCAG-3' \\
$5 p$ & Reverse & $5^{\prime}$ - TCCTCGTAGTCCAACACG-3' \\
\hline \multirow{2}{*}{ MAP3K2 } & Forward & $5^{\prime}$-GCTTACGGTCTCCTGTGAGTT-3' \\
& Reverse & $5^{\prime}$-AGGATTGTCTATGTCACTTCCCC- \\
& & $3^{\prime}$ \\
\hline \multirow{2}{*}{ G6 } & Forward & $5^{\prime}$-CTCGCTTCGGCAGCACA-3' \\
& Reverse & $5^{\prime}$-AACGCTTCACGAATTTGCGT-3' \\
\hline \multirow{2}{*}{ GAPDH } & Forward & $5^{\prime}$-GAGTCAACGGATTTGGTCGT-3' \\
& Reverse & $5^{\prime}$-TTGATTTTGGAGGGATCTCG-3' \\
\hline
\end{tabular}

and their corresponding negative control si-NC $\left(5^{\prime}\right.$ AAGACAUUGUGUGUCCGCCTT-3'), pcDNA-NR2F1AS1, and its negative control pcDNA, miR-493-5p mimic, and its negative control miR-NC were obtained from GenePharma (Shanghai, China). The transfections were carried out by Lipofectamine 3000. AGS and MKN-74 cells were cultured in an incubator at $37^{\circ} \mathrm{C}$ and $5 \% \mathrm{CO}_{2}$. After transfection for $48 \mathrm{~h}, \mathrm{AGS}$ and MKN-74 cells were collected for the functional experiments.

2.5. Western Blot Assay. The protein expression of MAP3K2 was detected by the Western blot assay. Cells in each group were collected to extract the total protein, and the protein concentration was detected by the BCA method. $30 \mu \mathrm{g}$ protein was electrophoresized by SDS-PAGE and then transferred to the PVDF membrane. Next, it was sealed with $5 \%$ skim milk for $2 \mathrm{~h}$ and added primary antibody (antiMAP3K2, $1: 1000$ ) at $4^{\circ} \mathrm{C}$ overnight. After washing with TBST, secondary antibody (goat-anti-mouse, $1: 1000$ ) was added to incubate for $1 \mathrm{~h}$. The gray values of each strip were measured by the gel imaging analysis system and Image J software.

2.6. Cell Proliferation. Cells were seeded in a 96-well plate with a cell density of $5 \times 10^{3}$ cells/well. After cultured at $37^{\circ} \mathrm{C}$ for 1-3 days, $10 \mu \mathrm{l}$ MTT ( $5 \mathrm{~g} / \mathrm{L}$ ) was added. After incubation for $4 \mathrm{~h}$, cells were added with $150 \mu \mathrm{l}$ dimethyl sulfoxide and incubated for $10 \mathrm{~min}$. Cell proliferation was indirectly reflected by measuring the OD at $490 \mathrm{~nm}$.

2.7. Cell Migration. AGS and MKN-74 cells were seeded in the upper chamber of the transwell chamber. We added $600 \mu \mathrm{l}$ Dulbecco's Modified Eagle Medium (DMEM) medium with $10 \%$ FBS to the lower chamber. The chamber was incubated in a $5 \% \mathrm{CO}_{2}$ incubator at $37^{\circ} \mathrm{C}$ for $24 \mathrm{~h}$. After washing with phosphate buffer saline (PBS), cells were fixed and stained by paraformaldehyde and $0.1 \%$ crystal violet. Finally, the number of migrated cells was measured by the microscope.

2.8. Cell Apoptosis. AGS and MKN-74 cells were centrifuged at $1000 \mathrm{r} / \mathrm{min}$ for $5 \mathrm{~min}$, and the supernatant was discarded. 
Then, cells were added with $5 \mu \mathrm{L}$ Annexin V-FITC and PI. Finally, cell apoptosis was detected by flow cytometry after incubation at room temperature in dark.

2.9. Dual-Luciferase Reporter Assay. NR2F1-AS1-WT, NR2F1-AS1-MUT, MAP3K2-WT, and MAP3K2-MUT were obtained from GenePharma (Shanghai, China). Cells were transfected with miR-493-5p mimic and miR-NC, respectively. After transfection for $48 \mathrm{~h}$, the luciferase activity was detected according to the requirements of the dual-luciferase reporter kit.

2.10. Statistical Analysis. The measurement data were presented as mean \pm SD. Data were analyzed by using SPSS 21.0 statistical software. The comparison between the two groups was analyzed by the $t$-test. The chi-square test was performed to compare the two groups. $P<0.05$ was the result of the statistical difference.

\section{Results}

3.1. NR2F1-AS1 and MAP3K2 Were Overexpressed in GC Tissues and Cells. To investigate the role of NR2F1-AS1 and MAP3K2 in the progression of GC, the expression levels of NR2F1-AS1 and MAP3K2 were assessed by RT-qPCR. Results reflected an upward trend of NEAT1 expression in GC tissues compared with control tissues (Figure 1(a)). Next, NR2F1-AS1 was obviously upregulated in GC cells (AGS, HGC-27, MKN-74, and SNU-5) compared with GES1 cells (Figure 1(b)). Next, we investigated the relationship between NEAT1 expression and overall survival rate. GC patients with high NR2F1-AS1 expression had a shorter survival time than those with low NR2F1-AS1 expression (Figure 1(c)). Moreover, MAP3K2 was identified as an ascending expression in GC tissues compared with control tissues (Figure 1(d)). Subsequently, MAP3K2 was highly expressed in GC cells compared to the normal cells (Figure 1(e)). Furthermore, there was a positive correlation between NR2F1-AS1 and MAP3K2 in GC tissues (Figure 1(f)). Therefore, NR2F1-AS1 and MAP3K2 may play a special role in the development of GC.

3.2. Knockdown of NR2F1-AS1 Repressed Cell Proliferation and Migration, While It Induced Cell Apoptosis of Cells. The expression of NR2F1-AS1 was significantly decreased when AGS and MKN-74 cells were transfected with NR2F1-AS1 si-RNA (Figure 2(a)). Then, the effect of NR2F1-AS1 on GC cell progression was detected by MTT, transwell assay, and FCM. As shown in Figures 2(b) and 2(c), after transfection with NR2F1-AS1 si-RNA, the OD value in GC cells decreased significantly. Compared with the control group, the migration ability of GC cells was obviously suppressed by NR2F1-AS1 knockdown (Figure 2(e)). In contrast, NR2F1-AS1 knockdown obviously accelerated the cell apoptosis rate of GC cells (Figure 2(d)).
3.3. Inhibition of MAP3K2 Suppressed Cell Proliferation and Migration and Enhanced Cell Apoptosis of GC Cells. In the same way, AGS and MKN-74 cells were transfected with MAP3K2 si-RNA. As shown in Figure 3(a), the protein expression of MAP3K2 was obviously reduced after cells were transfected with MAP3K2 si-RNA. Compared with the control group, MAP3K2 knockdown notably blocked cell proliferation in GC cells (Figures 3(b) and 3(c)). Similarly, MAP3K2 knockdown suppressed GC cell migration in cells compared with the control group (Figure 3(d)). As we expected, the cell apoptosis rate was ascended when GC cells were transfected with MAP3K2 knockdown (Figure 3(e)).

3.4. NR2F1-AS1 Targeted miR-493-5p, and MAP3K2 Was a Target Gene of miR-493-5p in GC Cells. LncRNAs have been reported to play their roles in human cancers by targeting miRNAs to regulate the downstream genes. In the current study, StarBase and TargetScan were used to predict the binding sequences between NR2F1-AS1, miR-493-5p, and MAP3K2 (Figures 4(a) and 4(b)). Then, we found that the luciferase activity of the NR2F1-AS1-WT group was notably declined after treatment with miR-493-5p mimic, while transfection of miR-493-5p mimic had no significant effect on the luciferase activity of the NR2F1-AS1-MUT group (Figures 4(c) and 4(d)). Next, RT-qPCR results displayed that the expression of miR-493-5p was increased in GC cells treated with NR2F1-AS1 knockdown, but declined when cells were treated with NR2F1-AS1 (Figures 4(e) and 4(f)). Similar experiments displayed that miR-493-5p mimic decreased the luciferase activity of MAP3K2-WT, but not MAP3K2-MUT (Figures 4(g) and 4(h)). In addition, the expression of MAP3K2 was notably descended by miR-493$5 p$ mimic, while it was increased by the miR-493-5p inhibitor (Figures 4(i) and 4(j)).

3.5. Downregulation of MAP3K2 Induced by miR-493-5p Could Be Recovered by Transfection with NR2F1-AS1 in GC Cells. We noticed that the protein expression of MAP3K2 was notably reduced by miR-493-5p mimic, while NR2F1AS1 retarded the inhibiting effect of miR-493-5p on MAP3K2 expression (Figures 5(a) and 5(b)). Therefore, NR2F1-AS1 regulated GC cell progression by inhibiting miR-493-5p to upregulate MAP3K2 expression.

\section{Discussion}

NR2F1-AS1 is verified to be aberrantly expressed in several human tumors and plays an important role in the development of tumors. NR2F1-AS1 was upregulated in hepatocellular carcinoma (HCC), and downregulated NR2F1AS1 was found to suppress cell growth and EMT progression and promote cell apoptosis of HCC cells [23]. Moreover, high NR2F1-AS1 expression was associated with poor OS, and silencing of NR2F1-AS1 blocked HCC cell hypoxiainduced glycolysis [24]. Furthermore, NR2F1-AS1 knockdown was reported to suppress cell invasion, migration, and cell growth, while it induced cell apoptosis in osteosarcoma cells by miR-483-3p and FOXA1 [25]. Whereas, the role of 


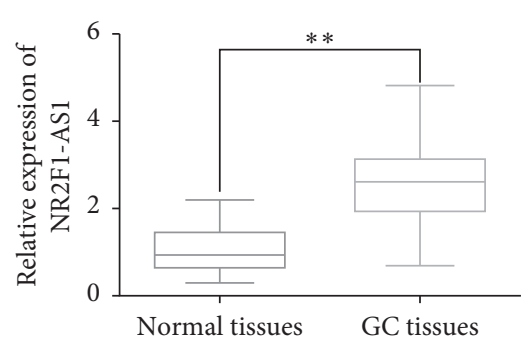

(a)

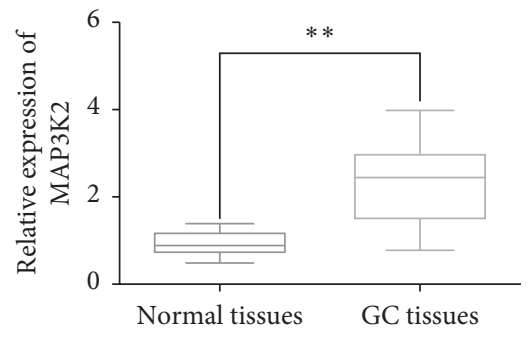

(d)

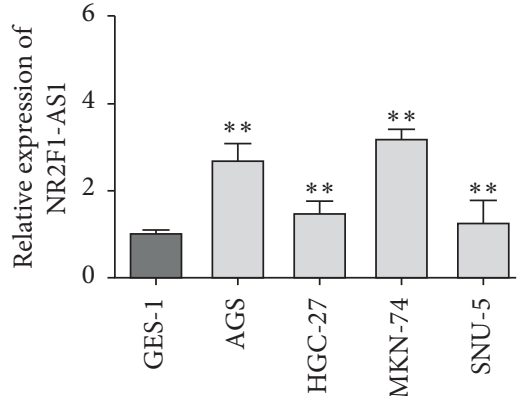

(b)

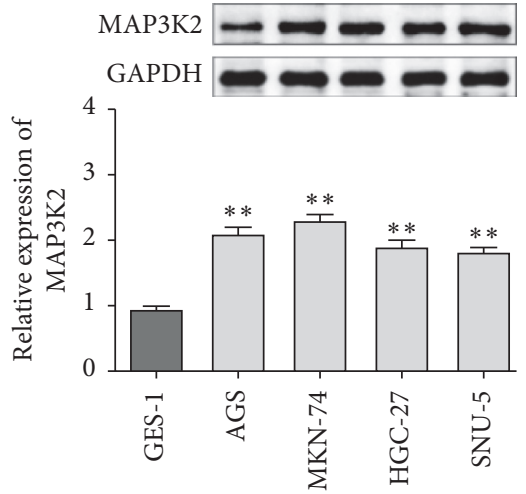

(e)

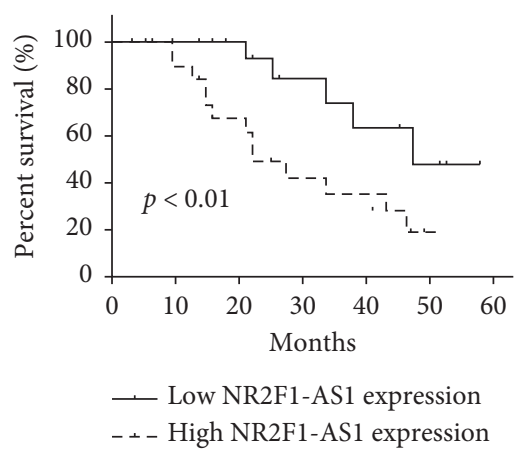

(c)

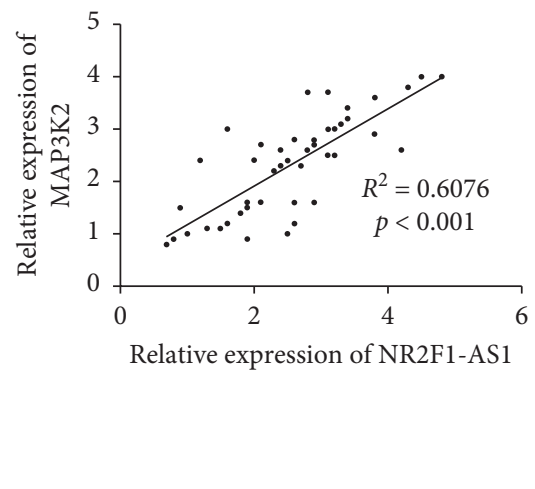

(f)

Figure 1: NR2F1-AS1 and MAP3K2 highly expressed in GC tissues and cells. (a, b) The expression of NR2F1-AS1 in 44 GC tissues and cell lines. (c) The relationship between NR2F1-AS1 and overall survival of GC patients. (d, e) The expression of NR2F1-AS1 in 44 GC tissues and cell lines. (f) The relationship between NR2F1-AS1 and MAP3K2 in GC tissues. ${ }^{* *} P<0.01$.

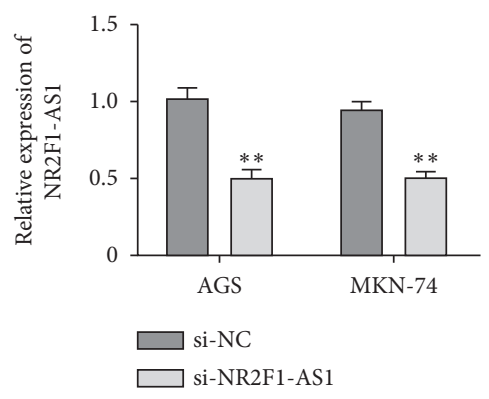

(a)

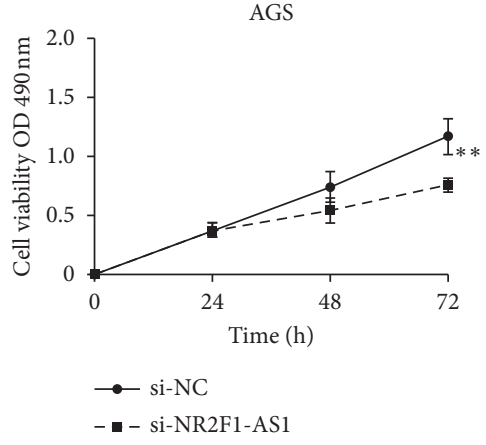

(b)

Figure 2: Continued. 


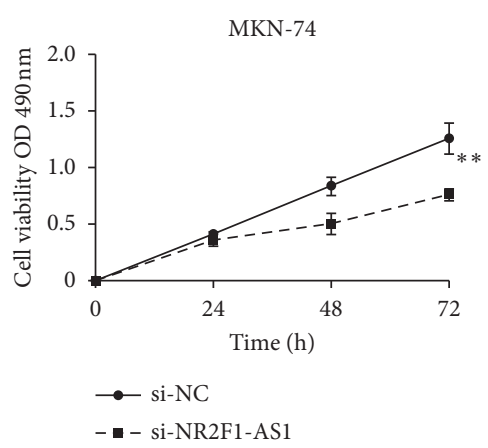

(c)

AGS
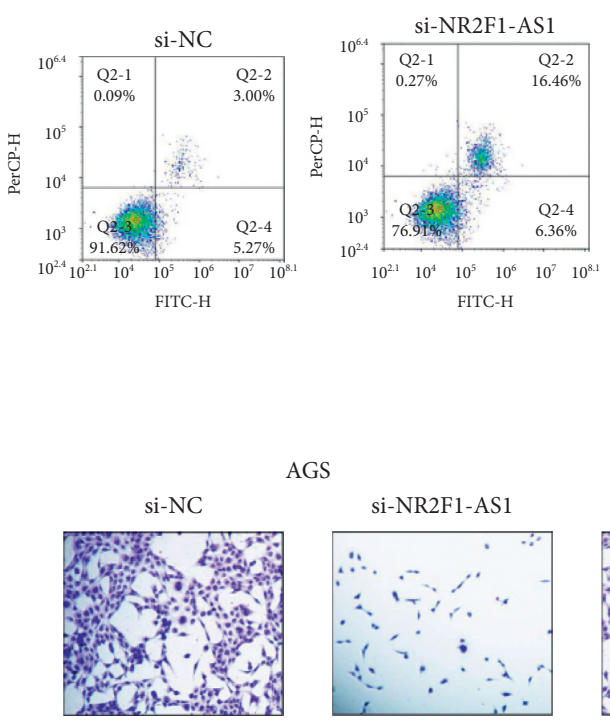

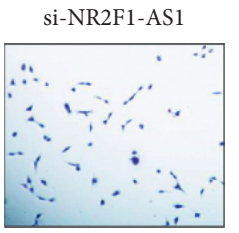

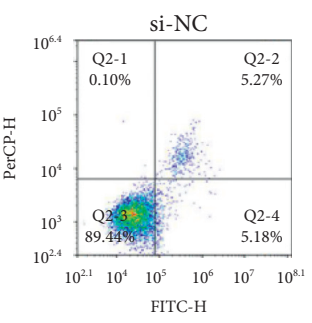

(d)
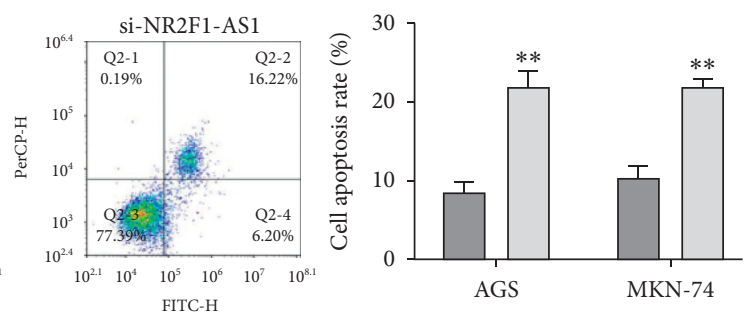

si-NC

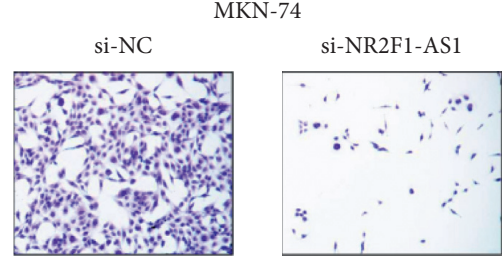

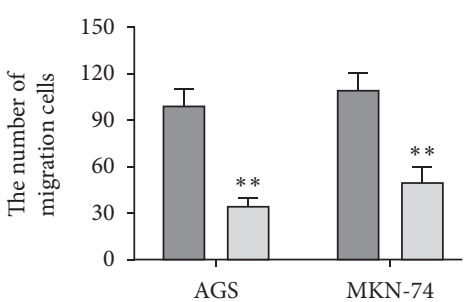

$\square$ si-NC

(e)

FIGURE 2: Knockdown of NR2F1-AS1 repressed cell proliferation and migration, while induced cell apoptosis of cells. (a) The expression of NR2F1-AS1 in AGS cells and MKN-74 cells transfected with NR2F1-AS1 si-RNA. (b, c) Cell proliferation in AGS cells and MKN-74 cells with si-NR2F1-AS1. (d) Cell apoptosis in AGS cells and MKN-74 cells with si-NR2F1-AS1. (e) Cell migration in AGS cells and MKN-74 cells with si-NR2F1-AS1 (scale bar $=100 \mu \mathrm{m})$. ${ }^{* *} P<0.01$.

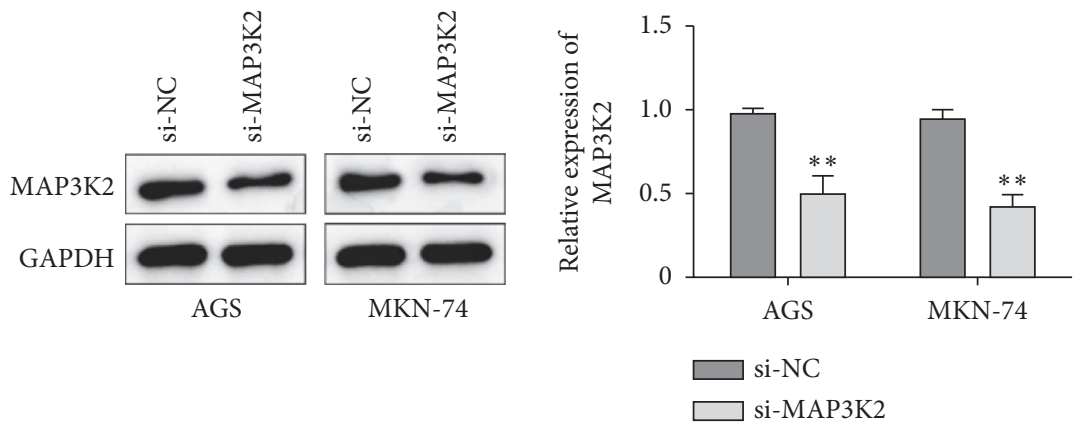

(a)

FIgURE 3: Continued. 


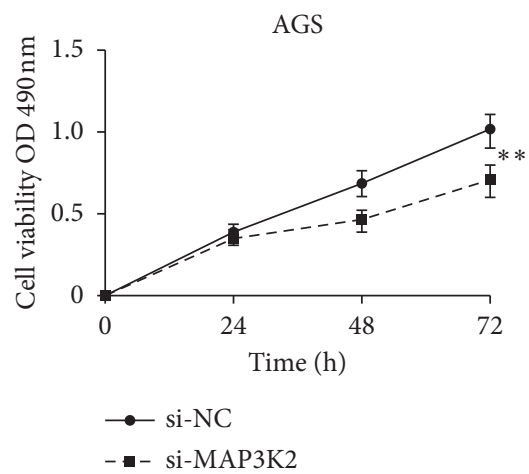

(b)

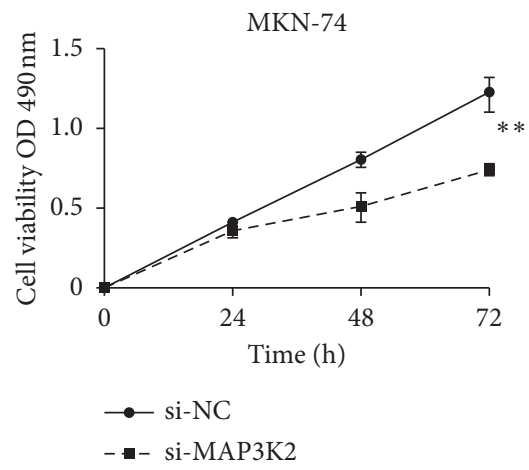

(c)

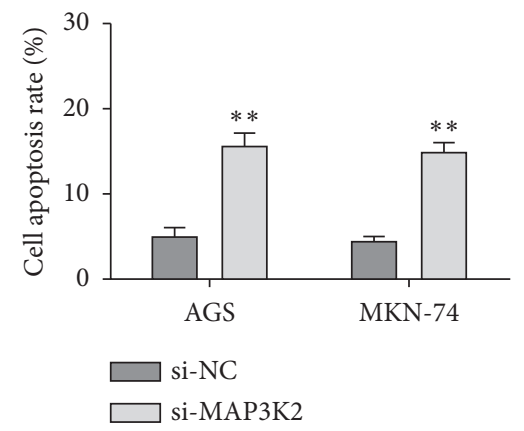

(d)

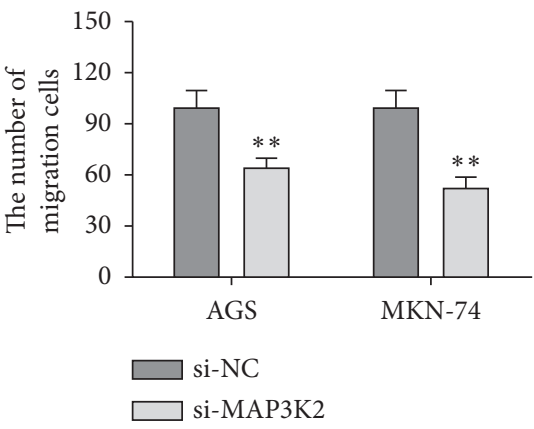

(e)

FIGURE 3: Inhibition of MAP3K2 suppressed cell proliferation and migration and enhanced cell apoptosis of GC cells. (a) The expression of MAP3K2 in AGS cells and MKN-74 cells with si-MAP3K2. (b, c) Cell proliferation in AGS cells and MKN-74 cells with si-MAP3K2. (d) Cell apoptosis in AGS cells and MKN-74 cells with si-MAP3K2. (e) Cell migration in AGS cells and MKN-74 cells with si-MAP3K2. ${ }^{* *} P<0.01$. 


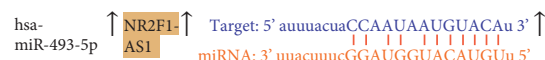

(a)

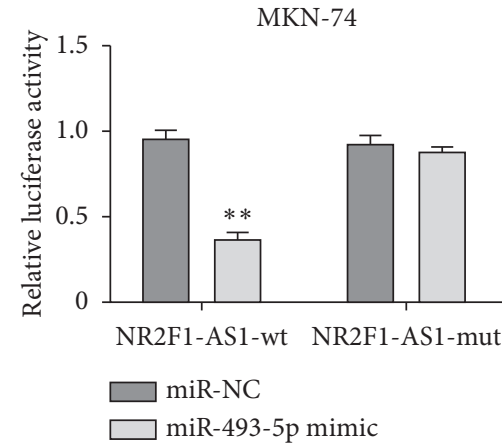

(d)

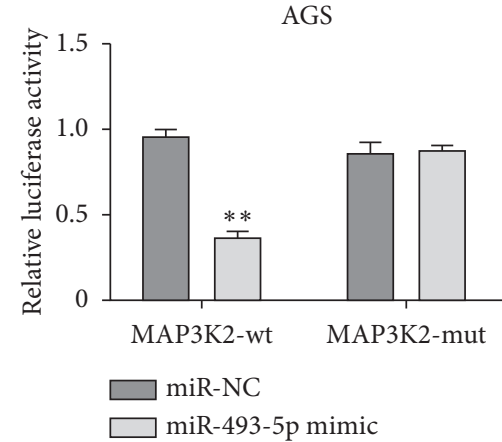

(g)
MAP3K2-wt 5 '-CCCUUUGACAUAAUAAUGUACAU-3' miR-493-5p 3'-UUACUUUCGGAUGGUACAUGUU-5' MAP3K2-mut 5'-CCCUUUGACAUAAUAUACAUGUU-3'

(b)

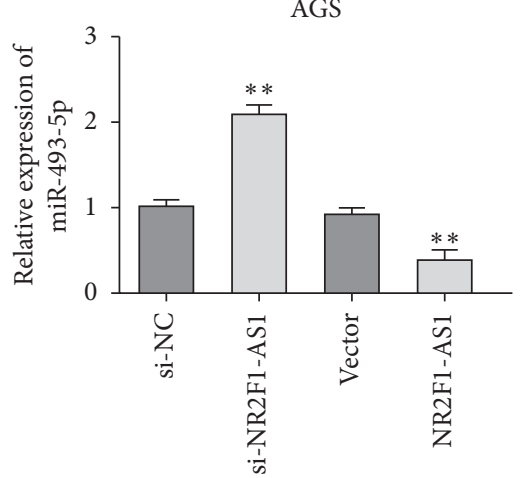

(e)

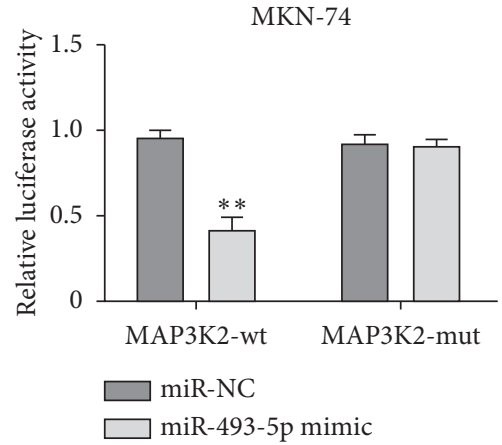

(h)

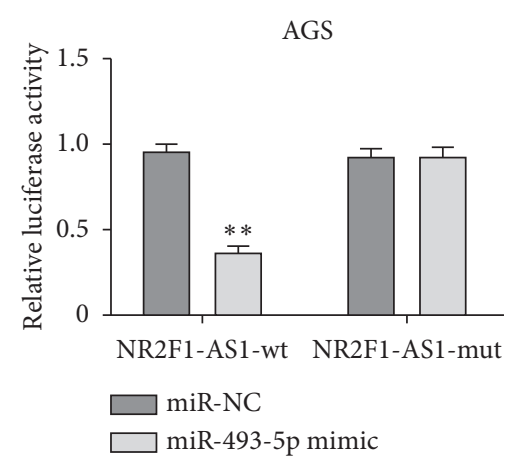

(c)

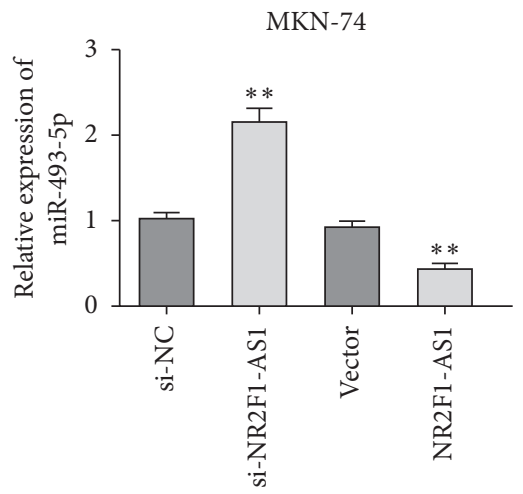

(f)

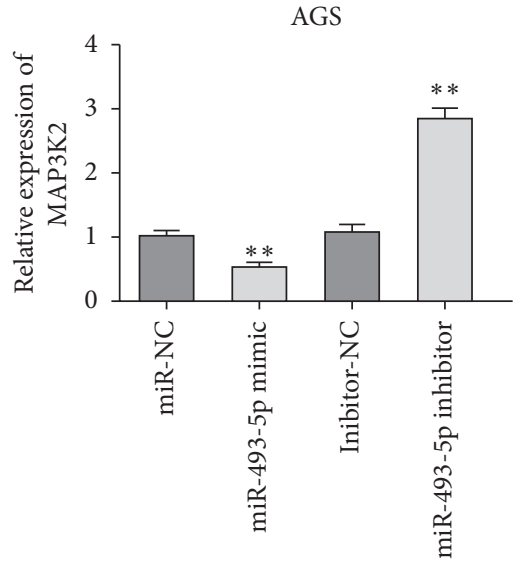

(i)

Figure 4: Continued. 


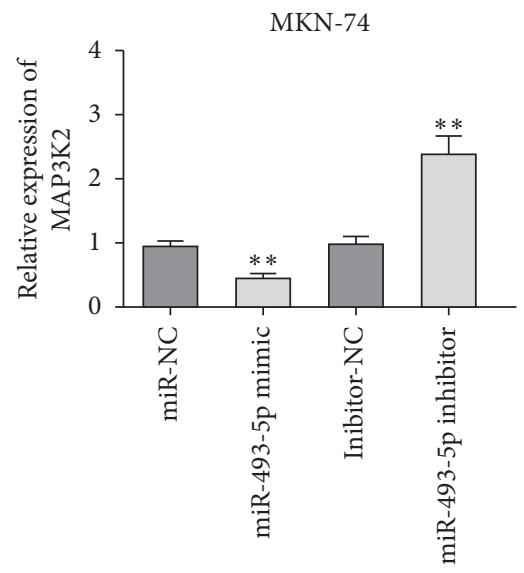

(j)

FIGURE 4: NR2F1-AS1 targeted miR-493-5p, while MAP3K2 was a target gene of miR-493-5p in GC cells. (a) The binding sites between NR2F1-AS1 and miR-493-5p. (b) The binding sites between MAP3K2 and miR-493-5p. (c, d) The effect of miR-493-5p on the luciferase activity of NR2F1-AS1-WT and NR2F1-AS1-MUT. (e, f) The expression of miR-493-5p in AGS cells and MKN-74 cells with si-NR2F1-AS1 or NR2F1-AS1. (g, h) The effect of miR-493-5p on the luciferase activity of MAP3K2-WT and MAP3K2-MUT. (i, j) The expression of MAP3K2 in AGS cells and MKN-74 cells with miR-493-5p mimic or miR-493-5p inhibitor. ${ }^{* *} P<0.01$.

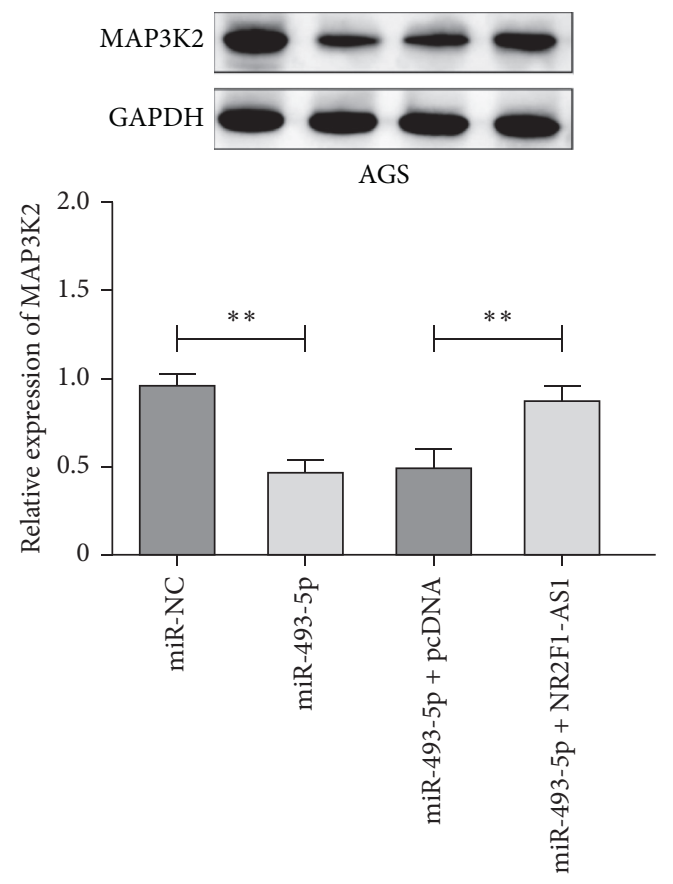

(a)

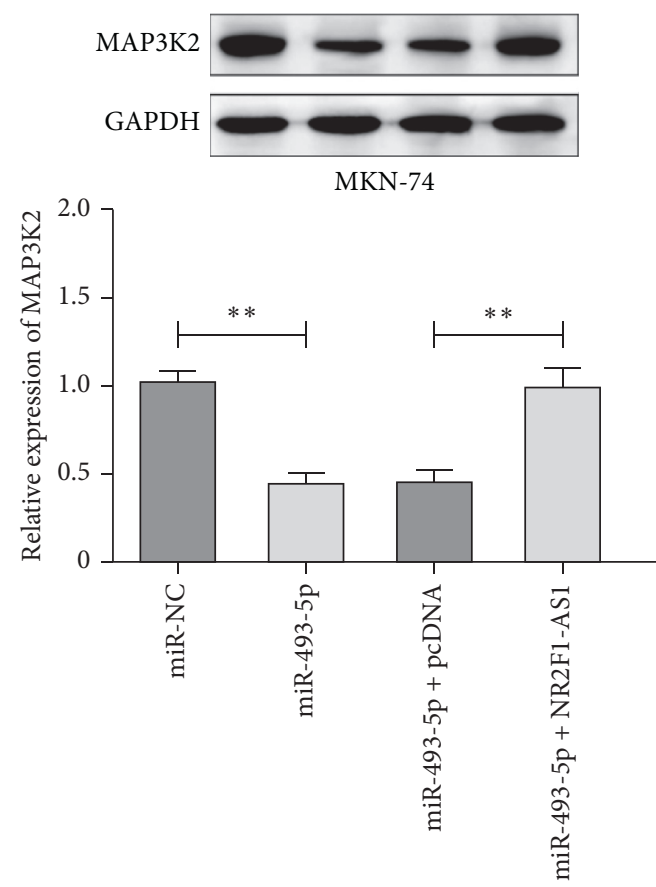

(b)

FIGURE 5: Downregulation of MAP3K2 induced by miR-493-5p could be recovered by transfection with NR2F1-AS1 in GC cells. (a) The expression of MAP3K2 in AGS cells with miR-493-5p or NR2F1-AS1. (b) The expression of MAP3K2 in MKN-74 cells with miR-493-5p or NR2F1-AS1. ${ }^{* *} P<0.01$.

NR2F1-AS1 in GC is not well-understood. In our experiment, we detected the expression level of NR2F1-AS1 in GC. Moreover, functional experiments were performed to measure the effect of NR2F1-AS1 on GC progression. Additionally, the possible carcinogenic mechanism of NR2F1AS1 in GC was discussed through molecular biology experiments.
We noticed that NR2F1-AS1 was notably overexpressed in GC tissues and cell lines. Our results were similar to previous reports. NR2F1-AS1 was highly expressed in melanoma, endometrial cancer, and cancer esophageal squamous cell carcinoma $[19,26,27]$. Furthermore, to detect the role of NR2F1-AS1 on GC progression, we knocked down the expression of NR2F1-AS1 in AGS and MKN-74 
cells. Our results displayed that NR2F1-AS1 knockdown dramatically blocked cell proliferation and migration, while it enhanced cell apoptosis of GC cells. Similarly, NR2F1-AS1 downregulation was found to inhibit cell growth, migration, invasion, and the EMT process in esophageal squamous cell carcinoma cells [26]. Contrary to our findings, NR2F1-AS1 was displayed to play as an antigene in colorectal cancer and cervical squamous cell carcinoma $[22,28]$.

LncRNAs were reported to play vital roles in tumor progression by sponging miRNAs to regulate downstream genes. In papillary thyroid carcinoma, NR2F1-AS1 acted as an oncogene by sponging miR-423-5p to upregulate SOX12 expression [29]. In the current study, miR-493-5p was a target of NR2F1-AS1, and MAP3K was a downstream gene of miR-493-5p. Mitogen-activated protein kinase 2 (MAP3K2) is a member of the MAP3K family with known roles in activating JNK and other kinases in the MAP pathway [30, 31]. MAP3K2 is reported to be necessary for the activation of ERK1/2 and MEK1/2 [32]. We found that MAP3K2 was significantly overexpressed in GC, and silence of MAP3K2 inhibited cell proliferation and migration, but facilitated cell apoptosis in GC cells. In line with our data, MAP3K2 was reported to be an oncogene in nonsmall cell lung cancer and triple-negative breast cancer $[33,34]$. Furthermore, MAP3K2 was significantly reduced by miR-493-5p mimic, while NR2F1-AS1 retarded the inhibitory effect of miR-493-5p. Finally, the network of NR2F1-AS1/miR-493-5p/MAP3K2 in GC progression was constructed.

\section{Conclusion}

In sum, NR2F1-AS1 was verified to be an oncogene in GC. Furthermore, we confirmed that NR2F1-AS1 regulated GC progression by sponging miR-493-5p to accelerate MAP3K2 expression. Therefore, our results suggested that NR2F1-AS1 might be a therapeutic target for patients with GC. However, the effect of NR2F1-AS1/miR-493-5p/MAP3K2 on the relevant pathways and the possible regulatory mechanism in the process of GC progression still need to be further studied.

\section{Data Availability}

The data used to support the findings of this study are available from the corresponding author upon request.

\section{Conflicts of Interest}

The authors declare that they have no conflicts of interest.

\section{Authors' Contributions}

Xiaobin Liao and Linbao Wen contributed equally to the article.

\section{References}

[1] F. Bray, J. Ferlay, I. Soerjomataram, R. L. Siegel, L. A. Torre, and A. Jemal, "Global cancer statistics 2018: GLOBOCAN estimates of incidence and mortality worldwide for 36 cancers in 185 countries," CA: A Cancer Journal for Clinicians, vol. 68, no. 6, pp. 394-424, 2018.

[2] M. Plummer, S. Franceschi, J. Vignat, D. Forman, and C. de Martel, "Global burden of gastric cancer attributable toHelicobacterpylori," International Journal of Cancer, vol. 136, no. 2, pp. 487-490, 2015.

[3] A. Ferro, V. Rosato, M. Rota et al., "Meat intake and risk of gastric cancer in the stomach cancer pooling (StoP) project," International Journal of Cancer, vol. 147, no. 1, pp. 45-55, 2020.

[4] H. Zafar, M. H. Kiani, F. Raza et al., "Design of enzyme decorated mucopermeating nanocarriers for eradication of H. pylori infection," Journal of Nanoparticle Research, vol. 22, no. 1, p. 4, 2020.

[5] F. Raza, H. Zafar, S Zhang et al., "Recent advances in cell membrane-derived biomimetic nanotechnology for cancer immunotherapy," Advanced Healthcare Materials, vol. 10, no. 6, Article ID e2002081, 2021.

[6] T. Hanyu, A. Wakai, T. Ishikawa, H. Ichikawa, H. Kameyama, and T. Wakai, "Carcinoma in the remnant stomach during long-term follow-up after distal gastrectomy for gastric cancer: analysis of cumulative incidence and associated risk factors," World Journal of Surgery, vol. 42, no. 3, pp. 782-787, 2018.

[7] L. Jiang, X. Gong, W. Liao, N. Lv, and R. Yan, "Molecular targeted treatment and drug delivery system for gastric cancer," Journal of Cancer Research and Clinical Oncology, vol. 147, no. 4, pp. 973-986, 2021.

[8] T. Seidlitz, Y.-T. Chen, H. Uhlemann et al., "Mouse models of human gastric cancer subtypes with stomach-specific CreERT2-mediated pathway alterations," Gastroenterology, vol. 157, no. 6, pp. 1599-1614, 2019.

[9] J. Zhou, J. Liu, H. Xing et al., "Implications of protein ubiquitination modulated by lncRNAs in gastrointestinal cancers," Biochemical Pharmacology, vol. 188, Article ID 114558, 2021.

[10] G. Militello, T. Weirick, D. John, C. Döring, S. Dimmeler, and S. Uchida, "Screening and validation of lncRNAs and circRNAs as miRNA sponges," Briefings in Bioinformatics, vol. 18, no. 5, pp. 780-788, 2017.

[11] K. Taniue and N. Akimitsu, "The functions and unique features of LncRNAs in cancer development and tumorigenesis," International Journal of Molecular Sciences, vol. 22, no. 2, p. 632, 2021.

[12] K.-T. Jin, J.-Y. Yao, X.-L. Fang, H. Di, and Y.-Y. Ma, "Roles of lncRNAs in cancer: focusing on angiogenesis," Life Sciences, vol. 252, Article ID 117647, 2020.

[13] H. Zhou, W. Shen, H. Zou, Q. Lv, and P. Shao, "Circulating exosomal long non-coding RNA H19 as a potential novel diagnostic and prognostic biomarker for gastric cancer," Journal of International Medical Research, vol. 48, no. 7, Article ID 300060520934297, 2020.

[14] J. Yan, Y. Zhang, Q. She et al., "Long noncoding RNA H19/ miR-675 Axis promotes gastric cancer via FADD/caspase 8/ caspase 3 signaling pathway," Cellular Physiology and Biochemistry, vol. 42, no. 6, pp. 2364-2376, 2017.

[15] G. Liu, T. Xiang, Q.-F. Wu, and W.-X. Wang, "Long noncoding RNA H19-derived miR-675 enhances proliferation and invasion via RUNX1 in gastric cancer cells," Oncology Research Featuring Preclinical and Clinical Cancer Therapeutics, vol. 23, no. 3, pp. 99-107, 2016.

[16] H. Feng, J. Zhang, Y. Shi, L. Wang, C. Zhang, and L. Wu, "Long noncoding RNA LINC-PINT is inhibited in gastric 
cancer and predicts poor survival," Journal of Cellular Biochemistry, vol. 120, no. 6, pp. 9594-9600, 2019.

[17] A. Sanchez Calle, T. Yamamoto, Y. Kawamura et al., "Long non-coding NR2F1-AS1 is associated with tumor recurrence in estrogen receptor-positive breast cancers," Molecular Oncology, vol. 14, no. 9, pp. 2271-2287, 2020.

[18] Q. Zhang, T. Li, Z. Wang, X. Kuang, N. Shao, and Y. Lin, "IncRNA NR2F1-AS1 promotes breast cancer angiogenesis through activating IGF-1/IGF-1R/ERK pathway," Journal of Cellular and Molecular Medicine, vol. 24, no. 14, pp. 82368247, 2020.

[19] L. Wang, S. Zhao, and Y. U. Mingxin, "LncRNA NR2F1-AS1 is involved in the progression of endometrial cancer by sponging miR-363 to target SOX4," Die Pharmazie, vol. 74, no. 5, pp. 295-300, 2019.

[20] P. Ren, H. Zhang, L. Chang, X. D. Hong, and L. Xing, "LncRNA NR2F1-AS1 promotes proliferation and metastasis of ESCC cells via regulating EMT," European Review for Medical and Pharmacological Sciences, vol. 24, no. 7, pp. 3686-3693, 2020.

[21] C. Zhang, S. Wu, R. Song, and C. Liu, "Long noncoding RNA NR2F1-AS1 promotes the malignancy of non-small cell lung cancer via sponging microRNA-493-5p and thereby increasing ITGB1 expression," Aging, vol. 13, no. 5, pp. 7660-7675, 2020.

[22] J. Wang, S. Dong, J. Zhang et al., "LncRNA NR2F1-AS1 regulates miR-371a-3p/TOB1 Axis to suppress proliferation of colorectal cancer cells," Cancer Biotherapy and Radiopharmaceuticals, vol. 35, no. 10, pp. 760-764, 2020.

[23] W.-C. Ji, G.-J. Bao, F.-W. Yang, L. Sun, and R. Han, "Role of lncRNA NR2F1-AS1 and lncRNA H19 genes in hepatocellular carcinoma and their effects on biological function of huh-7," Cancer Management and Research, vol. 13, pp. 941-951, 2021.

[24] X. Li, Y. Li, S. Bai, J. Zhang, Z. Liu, and J. Yang, "NR2F1-AS1/ miR-140/HK2 Axis regulates hypoxia-induced glycolysis and migration in hepatocellular carcinoma," Cancer Management and Research, vol. 13, pp. 427-437, 2021.

[25] S. Li, K. Zheng, Y. Pei, W. Wang, and X. Zhang, "Long noncoding RNA NR2F1-AS1 enhances the malignant properties of osteosarcoma by increasing forkhead box A1 expression via sponging of microRNA-483-3p," Aging, vol. 11, no. 23, pp. 11609-11623, 2019.

[26] Y. Zhang, A. Zheng, R. Xu et al., "NR2F1-induced NR2F1AS1 promotes esophageal squamous cell carcinoma progression via activating Hedgehog signaling pathway," Biochemical and Biophysical Research Communications, vol. 519, no. 3, pp. 497-504, 2019.

[27] M. Bai, Z. Z. Wu, Y. L. Huang, J. Ke, Q. Xu, and X. Wang, "STAT3 activates the transcription of lncRNA NR2F1-AS1 to promote the progression of melanoma via regulating the miR493-5p/GOLM1 axis," The Journal of Gene Medicine, vol. 23, no. 7, Article ID e3338, 2021.

[28] J. Peng, F. Hou, W. Zhu, J. Li, and Z. Teng, "IncRNA NR2F1AS1 regulates miR-17/SIK1 Axis to suppress the invasion and migration of cervical squamous cell carcinoma cells," $R e$ productive Sciences, vol. 27, no. 7, pp. 1534-1539, 2020.

[29] C. Yang, Z. Liu, X. Chang et al., "NR2F1-AS1 regulated miR423-5p/SOX12 to promote proliferation and invasion of papillary thyroid carcinoma," Journal of Cellular Biochemistry, vol. 121, no. 2, pp. 2009-2018, 2020.

[30] B. Su, J. Cheng, J. Yang, and Z. Guo, "MEKK2 is required for T-cell receptor signals in JNK activation and interleukin-2 gene expression," Journal of Biological Chemistry, vol. 276, no. 18, pp. 14784-14790, 2001.
[31] C.-D. Ji, Y.-X. Wang, D.-F. Xiang et al., "Kir2.1 interaction with Stk38 promotes invasion and metastasis of human gastric cancer by enhancing MEKK2-MEK1/2-ERK1/2 signaling," Cancer Research, vol. 78, no. 11, pp. 3041-3053, 2018.

[32] P. Colón-Bolea and P. Crespo, "Lysine methylation in cancer: SMYD3-MAP3K2 teaches us new lessons in the Ras-ERK pathway," BioEssays, vol. 36, no. 12, pp. 1162-1169, 2014.

[33] T. Huang, K. She, G. Peng et al., "MicroRNA-186 suppresses cell proliferation and metastasis through targeting MAP3K2 in non-small cell lung cancer," International Journal of Oncology, vol. 49, no. 4, pp. 1437-1444, 2016.

[34] J. Wu, W.-Z. Li, M.-L. Huang et al., "Regulation of cancerous progression and epithelial-mesenchymal transition by miR$34 \mathrm{c}-3 \mathrm{p}$ via modulation of MAP3K2 signaling in triple-negative breast cancer cells," Biochemical and Biophysical Research Communications, vol. 483, no. 1, pp. 10-16, 2017. 